\title{
Sensitivity Study of Nuclear Reactions Influencing Photo- spheric Radius Expansion X-Ray Bursts
}

\author{
Yi Hua Lam ${ }^{1,2, *}$, Alexander Heger ${ }^{3,4,5,6, * *}$, Zac Johnston ${ }^{6}$, and Adelle Jane Goodwin ${ }^{7}$ \\ ${ }^{1}$ Institute of Modern Physics, Chinese Academy of Sciences, Lanzhou 730000, China \\ ${ }^{2}$ School of Nuclear Science and Technology, University of Chinese Academy of Sciences, Beijing 100049, China \\ ${ }^{3}$ School of Physics and Astronomy, Monash University, Victoria 3800, Australia \\ ${ }^{4}$ OzGrav-Monash - MOCA, School of Physics and Astronomy, Monash University, VIC 3800, Australia \\ ${ }^{5}$ Center of Excellence for Astrophysics in Three Dimensions (ASTRO-3D), Australia \\ ${ }^{6}$ Joint Institute for Nuclear Astrophysics, Michigan State University, East Lansing, MI 48824, USA \\ ${ }^{7}$ International Centre for Radio Astronomy Research - Curtin University, Perth, WA 6845, Australia
}

\begin{abstract}
Up to now, more than 62 of the $115 \mathrm{X}$-ray sources of low-mass-X-ray binaries have been identified as photospheric radius expansion (PRE) bursters [1]. Galloway and collaborators expect more PRE bursters in their near future analysis [2]. Although more than half of the discovered X-ray sources are PRE bursters, the bursting mechanism of PRE burster is still not adequately understood. This is because of the complicated hydrodynamics and variable accretion rates. An example is the accretion-powered millisecond pulsar SAX J1808.4-3658 [3, 4] that powered up the brightest Type-I X-ray burst (XRB) recorded by NICER in recent history [5]. The first 1D multi-zone model of SAX J1808.4-3658 was recently constructed [6, 7]. The pioneering model offers a first concurrent and direct comparison with the observed light curves, fluences, and recurrence times. With the three observables, a comparison between theory and observations could be more sensitive than the previous studies of the clocked burster and post-processing models. We perform a sensitivity study on $(\alpha, \mathrm{p}),(\alpha, \gamma),(\mathrm{p}, \alpha)$, and $(\mathrm{p}, \gamma)$ reactions with a total up to $\sim 1,500$ reactions. Our current result indicates that the observables are more sensitive to the competition between the reactions involving alpha-capture, e.g., the ${ }^{22} \operatorname{Mg}(\alpha, p)$ and ${ }^{22} \mathrm{Mg}(\mathrm{p}, \gamma)$ reactions competing at the ${ }^{22} \mathrm{Mg}$ branch point [8].
\end{abstract}

Generally, due to less extensive rapid-proton capture (rp-) process but mainly powered by alpha-proton ( $\alpha$ p-) process, the light curves of PRE bursts commence with rapid onsets $(\lesssim 1 \mathrm{~s})$, extend with wide plateau $(\approx 10 \mathrm{~s})$, and end with short burst tail $(\lesssim 30 \mathrm{~s})$ [9-11]. A close match of modeled and observed PRE burst permit us to probe the complicated burst mechanism of PRE bursters. Johnston et al. [6] recently constructed the first PRE burst model quantitatively describing the accretion-powered millisecond pulsar SAX J1808.4-3658, whereas the other simplified model built by Goodwin et al. [7] provides an intuitive grasp of the observables of SAX J1808.4-3658 burster. The host neutron star of this PRE burster spins up to millisecond periods by the accretion of stellar matter from a low-mass companion star in its X-ray binary system [3]. The essential properties of an epoch of this PRE burster includes the burst light curves, fluences (integral of flux over time), and recurrence times. This PRE burster offers

\footnotetext{
*e-mail: lamyihua@impcas.ac.cn

**e-mail: alexander.heger@monash.edu
} 
more concurrent observables for us to compare with models. Therefore, we expect the comparison of these PRE-burster observables is more sensitive than merely the comparison of light curves and recurrence times, e.g., the comparison of light curves and recurrence times of the GS 1826-24 clocked burster. Recently, only the PRE models were used to quantitatively match with these three observables. Table 1 shows the comparison of available models, their characteristics, and XRB observables that had been studied with published results.

Table 1. XRB models and the respective observables that have been studied.

\begin{tabular}{|c|c|c|c|c|c|}
\hline OD One-zone post-processing & No & Yes & - & - & - \\
\hline 1D Multi-zone GS 1826-24 [12, 13] & Yes & Yes & Yes & Yes & - \\
\hline 1D Multi-zone SAX J1808.4-3658 $[6,7]$ & Yes & Yes & Yes & Yes & Yes \\
\hline
\end{tabular}

Concurrently comparing light curves, fluences, and recurrence times permits us to scrutinize the constructed XRB models, e.g., the assumed hydrodynamics, to simulate and to study the extreme astrophysical environment. Moreover, the sensitivity study on these three observables helps us to identify important reactions, and then to constrain and to improve the pioneer PRE XRB model, e.g., the $\mathrm{H} / \mathrm{He} /$ metaliticiy ratios and accretion rate. With a more constrained PRE XRB model, this opens new opportunities for us to study neutron-star compactness, mass, radius, and cooling processes of SAX J1808.4-3658 burster of which the host neutron star is extremely cold, exhibiting rapid and active cooling processes [14]. In additions, the hydrodynamic data generated from an 1D multi-zone SAX J1808.4-3658 XRB model could be beneficial for one-zone post-processing and one-zone models to obtain the nuclear energy generation (XRB flux) consistent with the input hydrodynamic snapshots.

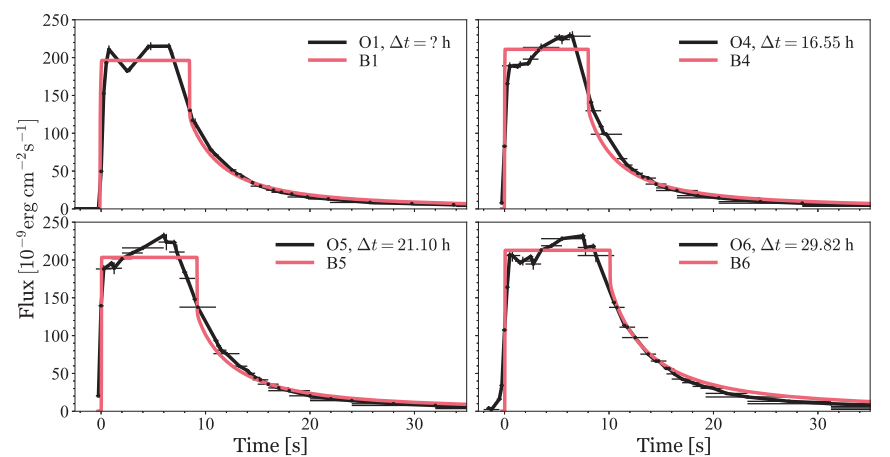

Figure 1 shows the modeled and observed PRE burst light curves of SAX J1808.4-3658. We reproduce the observed PRE bursts of SAX J1808.4-3658 (Epoch Oct. 2002) using Johnston et al. [6] and Goodwin et al. [7] models with Non-SMOKeR ${ }^{22} \operatorname{Mg}(\alpha$, p) reaction rate (yellow diamonds and purple stars in Fig. 2). Nevertheless, with implementing the most updated ${ }^{22} \mathrm{Mg}(\alpha, \mathrm{p})$ reaction rate experimentally deduced by $\mathrm{Hu}$ et al. [8] with the lowest uncertainty compared to all currently available ${ }^{22} \operatorname{Mg}(\alpha, \mathrm{p})$ rates, an extra burst is produced and the overall recurrence times of all PRE bursts are shifted (blue open squares in Fig. 2). This most updated ${ }^{22} \operatorname{Mg}(\alpha, \mathrm{p})$ rate is in fact almost one order of magnitude lower than Non-SMOKER ${ }^{22} \operatorname{Mg}(\alpha, \mathrm{p})$ rate. The primary reason for producing an extra burst is due to the competition between the ${ }^{22} \mathrm{Mg}(\alpha, \mathrm{p})$ and ${ }^{22} \mathrm{Mg}(\mathrm{p}, \gamma)$ reactions at the ${ }^{22} \mathrm{Mg}$ branch point within the accreting envelope maximum temperature range of PRE burst which lasts for around $10 \mathrm{~s}$ and is $1 / 3$ of the duration of a PRE burst. The process of restoring the PRE model permits us to constrain the mass 


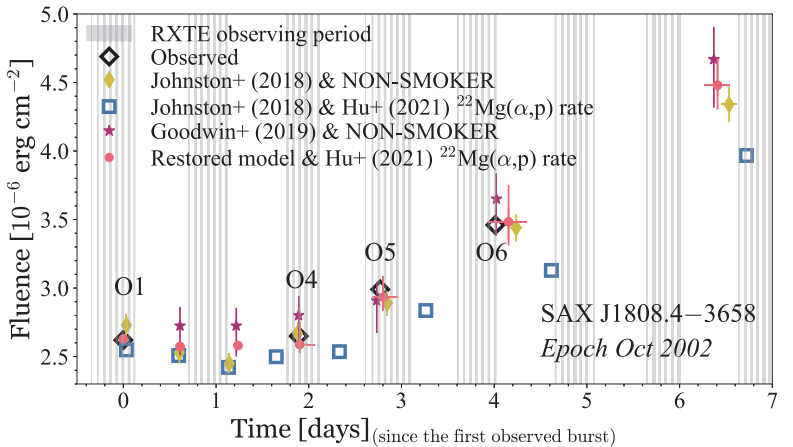

Figure 2. The bursts' fluences and times of the SAX J1808.4-3658 PRE burster, recorded by the Rossi X-ray Timing Explorer (RXTE) [1], based on Johnston et al. [6] and Goodwin et al. [7] models with NoN-SMOKER ${ }^{22} \operatorname{Mg}(\alpha, \mathrm{p})$ reaction rate, and the restored Johnston et al. model with Hu et al. ${ }^{22} \operatorname{Mg}(\alpha, \mathrm{p})$ reaction rate [8]. The light curves of observed (and modeled) bursts O1 (B1), O4 (B4), O5 (B5), and O6 (B6) are plotted in Fig. 1.

fractions of $\mathrm{H}$ and $\mathrm{He}$ in the accreting envelope of the PRE model to be $X_{\mathrm{H}}=41.3 \pm 0.3 \%$ and $X_{\mathrm{He}}=56.7 \pm 0.3 \%$. We find that the comparison of fluences-recurrence times due to a varied selected reaction is more sensitive than merely comparing the light curves of XRB, e.g., the light curve of GS 1826-24 clocked burster. This finding motivates us to explore the sensitivity of $\sim 1,500$ reactions along the nuclear reaction flow path during an episode of PRE bursts. These $\sim 1,500$ reactions consist of $(\alpha, \mathrm{p}),(\alpha, \gamma),(\mathrm{p}, \alpha)$, and $(\mathrm{p}, \gamma)$ reactions.

Our preliminary results indicate that these three observables are more sensitive to the competition between the reactions involving alpha-capture, e.g., the ${ }^{22} \operatorname{Mg}(\alpha, \mathrm{p})$ and ${ }^{22} \operatorname{Mg}(\mathrm{p}, \gamma)$ as shown above. Contrarily to the influence of ${ }^{22} \operatorname{Mg}(\alpha, \mathrm{p})$ reaction, if the ${ }^{34} \operatorname{Ar}(\alpha, \mathrm{p})$ reaction is one order of magnitude lower than its NoN-SMOKER counterpart, the PRE model produces one PRE burst fewer than the model implementing NoN-SMOKER ${ }^{34} \operatorname{Ar}(\alpha, \mathrm{p})$ rate. We anticipate that a list of important reactions of PRE burst is different from what was found by Cyburt et al. [15] based on the GS 1826-24 model. A set of more precisely determined important reaction rates definitely allows us to further constrain the pioneering PRE models. Further results will be made available and published elsewhere [16].

This work was financially supported by the Strategic Priority Research Program of Chinese Academy of Sciences (CAS, Grant Nos. XDB34000000) and National Natural Science Foundation of China (No. 11775277), the Chinese Academy of Sciences President's International Fellowship Initiative (No. 2019FYM0002), the Australian Research Council Centre of Excellence for Gravitational Wave Discovery (OzGrav, No. CE170100004) and for All Sky Astrophysics in 3 Dimensions (ASTRO 3D, No. CE170100013), Australian Research Council's Discovery Projects funding scheme (DP200102471). We are appreciative of the computing resource provided by the Institute of Physics (PHYS_T3 cluster) and the ASGC (Academia Sinica Grid-computing Center) Distributed Cloud resources (QDR4 and FDR5 clusters) of Academia Sinica, Taiwan.

\section{References}

[1] D. K. Galloway, J. in 't Zand, J. Chenevez et al., Astrophys. J. Suppl. 249, 32 (2020).

[2] D. K. Galloway private communication.

[3] R. Wijnands, M. van der Klis, Nature 394, 344 (1998).

[4] D. Chakrabarty, E. Morgan, M. Muno et al., Nature 424, 42 (2003)

[5] P. Bult, G. K. Jaisawal, T. Güver et al., Astrophys. J. Lett. 885, L1 (2019).

[6] Z. Johnston, A. Heger, D. K. Galloway, Mon. Not. R. Astron. Soc. 477, 2112 (2018).

[7] A. J. Goodwin, D. K. Galloway, A. Heger et al., Mon. Not. R. Astron. Soc. 490, 2228 (2019).

[8] J. Hu, H. Yamaguchi, Y. H. Lam et al., Phys. Rev. Lett. 127, 172701 (2021).

[9] W. H. G. Lewin, W. D. Vacca, E. M. Basinska, Astrophys. J. 277, L57 (1984).

[10] Y. Tawara, T. Kii, S. Hayakawa et al., Astrophys. J. 276, L41 (1984).

[11] T. Strohmayer, L. Bildsten, New Views of Thermonuclear Bursts in Compact Stellar X-ray Sources (Cambridge University Press, Cambridge, UK, 2010) p. 113.

[12] A. Heger, A. Cumming, D. K. Galloway et al., Astrophys. J. Lett. 671, L141 (2007).

[13] B. Paxton, P. Marchant, J. Schwab et al., Astrophys. J. Suppl. Ser. 220, 15 (2015).

[14] C. O. Heinke, P. G. Jonker, R. Wijnands et al., Astrophys. J. 691, 1035 (2009).

[15] R. H. Cyburt, A. M. Amthor, A. Heger et al., Astrophys. J. 830, 55 (2016).

[16] Y. H. Lam, A. Heger, Z. Johnston, A. J. Goodwin et al., in preparation. 